\title{
Stage 0 Low-Grade Appendiceal Mucinous Neoplasm (LAMN) AJCC v8
}

National Cancer Institute

\section{Source}

National Cancer Institute. Stage O Low-Grade Appendiceal Mucinous Neoplasm (LAMN)

A/CC v8. NCl Thesaurus. Code C134119.

Stage 0 includes: Tis(LAMN), N0, MO. T is(LAMN): Low-grade appendiceal mucinous neoplasm confined by the muscularis propria. Acellular mucin or mucinous epithelium may invade into the muscularis propria. T1 and T2 are not applicable to LAMN. Acellular mucin or mucinous epithelium that extends into the subserosa or serosa should be classified as T3 or T4a, respectively. N0: No regional lymph node metastasis. M0: No distant metastasis. (AJCC 8th ed.) 